\title{
Ejaculation-sparing thulium laser enucleation of the prostate (ES-ThuLEP): outcomes on a large cohort
}

\author{
Giorgio Bozzini ${ }^{1} \odot$ - Lorenzo Berti ${ }^{1,2} \cdot$ Matteo Maltagliati $^{1,2}$. Umberto Besana ${ }^{1} \cdot$ Alberto Calori $^{1} \cdot$ Alexander Müller $^{3}$. \\ Maria Chiara Sighinolfi ${ }^{2}$ Salvatore Micali $^{2} \cdot$ Antonio Luigi Pastore $^{4} \cdot$ Rodrigo Ledezma $^{5} \cdot$ Paolo Broggini $^{6}$. \\ Bernardo Rocco ${ }^{2}$. Carlo Buizza ${ }^{1}$
}

Received: 22 June 2020 / Accepted: 5 September 2020

(c) Springer-Verlag GmbH Germany, part of Springer Nature 2020

\begin{abstract}
Purpose To assess the effects of a new ejaculation-sparing thulium laser enucleation of the prostate (ES-ThuLEP) technique on sexual functions and micturition, in patients with lower urinary tract symptoms secondary to benign prostatic hyperplasia (BPH) and to evaluate how the surgical technique of ES-ThuLEP can lead to ejaculation preservation.

Methods A prospective study was carried out between January 2015 and January 2018 on patients with surgical indication for BPH, who wished to preserve ejaculation. The patients were treated with ES-ThuLEP and were evaluated before and 3 and 6 months after surgery. Three validated questionnaires (ICIQ-MLUTSsex, IIEF-5 and IPSS) were used to assess changes in ejaculation, erectile function and urinary symptoms. Uroflowmetry (Qmax and Qavg), post-void residual volume and voided volume were also evaluated, to assess micturition improvement. Patients with moderate to severe erectile dysfunction were excluded. Statistical analysis was performed with the Student's $t$ test, Chi-square test and logistic regression analysis. Results Two hundred and eighty three patients were enrolled. Ejaculation was spared in 203 and 219 patients at 3 and 6 months after surgery. No significant differences were observed between erectile function before and after surgery: baseline IIEF-5 $=16.2 \pm 4.47$ vs $16.7 \pm 2.9(p=0.419)$ and $17.7 \pm 3.2(p=0.410)$ at 3 and 6 months. Significant improvement in urinary symptoms was achieved: baseline IPSS $=19.4 \pm 7.24$ vs $5.8 \pm 4.3(p=0.032)$ and $3.9 \pm 4.1(p=0.029)$ at 3 and 6 months.

Conclusion ES-ThuLEP effectively preserved ejaculation in over two thirds of the patients without compromising micturition improvement or erectile function. ES-ThuLEP could be a valid treatment option for BPH in young and sexually active men.
\end{abstract}

Keywords Ejaculation $\cdot$ Ejaculatory dysfunction $\cdot$ Ejaculation sparing $\cdot$ Benign prostatic hyperplasia $\cdot$ Lower urinary tract symptoms · Thulium laser · ThuLEP · Endoscopic enucleation of the prostate

\section{Introduction}

Giorgio Bozzini

gioboz@yahoo.it

1 Department of Urology, ASST Valle Olona, Ospedale di Busto Arsizio (VA), Busto Arsizio, Italy

2 Department of Urology, Università degli Studi di Modena e Reggio Emilia (MO), Modena, Italy

3 Department of Urology, Spital Limmattal, Schlieren, Switzerland

4 Department of Urology, Sapienza Università di Roma, Latina, Italy

5 Department of Urology, Hospital Clìnico Universidad de Chile, Santiago, Chile

6 Department of Urology, Clinica Sant'Anna, Lugano, Switzerland
Benign prostatic hyperplasia (BPH) is a common condition in aging men, which leads to lower urinary tract symptoms (LUTS) and sexual dysfunction, negatively impacting on the patients' quality of life [1,2]. Despite allowing relief from LUTS, classical medical and surgical treatments for $\mathrm{BPH}$ have always been burdened by important consequences on the patients' ejaculatory function. Over the past three decades research has focused on the developement of new surgical strategies to reduce morbidity and complications of conventional surgical procedures, such as transurethral resection of the prostate (TURP) and open prostatectomy (OP), but in most cases overlooking the impact on the patients' ejaculatory function. Thanks to the advent of laser technologies, endoscopic enucleation of the prostate (EEP) 
techniques have been developed [3, 4]. These mimick an OP, which is achieved endoscopically, using a laser, or a bipolar resector to enucleate the adenoma, imitating the use of the finger during OP, but maintining minimal invasiveness of TURP. Holmium laser enucleation of the prostate (HoLEP) [5] and thulium laser enucleation of the prostate (ThuLEP) [6] are the most demanded laser EEP techniques (LEEP). Both determine excellent resolution of LUTS and improvement in voiding parameters, comparable to those of TURP and OP, but with reduced morbidity, catheterization time and hospital stay. Moreover, they allow to treat prostates of virtually all sizes [7-10]. Reasons for which, EAU Guidelines reccomend to resort to OP only in the abscence of an EEP system [11]. Despite the well documented advantages of LEEP techniques over TURP and OP, they have not been able to overcome postoperative ejaculatory dysfunction (EjD) $[12,13]$.

The only reccomended ejaculation-sparing (ES) procedure today [11], is the prostatic urethral lift, which consists in the positioning of suture-based implants under cystoscopic guidance, to compress the lateral lobes [14]. Despite being a valid treatment option, especially in elderly and frail patients [15] and despite excellent outcomes on ejaculation, it does not achieve the same micturition improvement of TURP [16]. Moreover, it is only reccomended for small prostates $(<70 \mathrm{cc})$ and it does not remove the hyperplastic tissue. Other novel, minimally invasive, non-ablative surgical techniques such as Aquablation, Rezum, and prostatic artery embolization, despite presenting more favourable outcomes on ejaculation compared to TURP, are still considered "under investigation" by EAU Guidelines, due to the lack of randomized controlled trials, with a long-term follow-up, investigating their safety and efficacy $[11,17]$.

Before the ' $90 \mathrm{~s}$ ejaculation was thought to be a 4-phase process [18], which consisted of:

1. Erection.

2. Emission of semen into the prostatic urethra, closed between the internal sphincter (bladder neck) and the external sphincter.

3. Formation of a pressure chamber inside the prostatic urethra.

4. Expulsion of semen through the external sphincter.

According to this theory, loss of ejaculation after endoscopic surgery was and is still attributed by many to the removal of the internal sphincter, causing retrograde ejaculation [19].

However, evidence on ejaculation physiology has demonstrated the importance of the tissues surrounding the veru montanum, rather than the bladder neck, for outward ejaculation [20]. Dynamic transrectal ultrasonography (TRUS) has shown how, just before ejaculation, the veru montanum undergoes a caudal shift, making contact with the anterior wall of the urethra, allowing the antegrade progression of semen, which is emitted into the inframontanal urethra and expelled, almost simoultaneously, through the external sphincter [21]. No accumulation of semen inside the prostatic urethra, and therefore, generation of a high pressure area was demonstrated. A fundamental role in this process is played by the musculus ejaculatorious, a longitudinal strain of muscle fibres, which originate from around the ejaculatory ducts and extend caudally in the urethral crest, inserting below the urethral sphincter. Contraction of the musculus ejaculatorious could be responsible not only for the emission of semen from the ejaculatory ducts into the prostatic urethra, but also for the correct movement of the veru montanum during ejaculation [21, 22].

Based on these findings, supramontanal and paracollicular tissue-sparing surgical techniques have been developed. ES techniques have been applied to TURP and photoselective vaporization of the prostate (PVP) with encouraging results [23-25]. However, TURP and PVP are currently reccomended only for the treatment of small to intermediate size prostates [11]. Considering that LEEP techniques have already proved to be size indipendent $[9,10]$, we decided to modify classical ThuLEP surgery, by sparing $1.5 \mathrm{~cm}$ of tissue above the veru montanum and two hills of tissue at the apex of the lateral lobes, to evaluate whether such approach is truly effective in maintaining ejaculation, without compromising micturition improvement.

\section{Materials and methods}

\section{Patients' selection}

A prospective study was carried out between January 2015 and January 2018 on patients suffering from LUTS secondary to BPH, with surgical indication according to EAU Guidelines [11], who wished to preserve ejaculation. All the patients who met the inclusion criteria were enrolled and treated with ES-ThuLEP. Patients $>75$ years of age, or with moderate to severe erectile dysfunction (IIEF-5 $<12$ ), or who had undergone previous abdominal surgery, or open/ endoscopic surgery for BPH were excluded. Patients were assessed with a physical and digital transrectal examination, prostate specific antigen (PSA) levels, TRUS, suprapubic ultrasonography, to assess post-void residual volume (PVR), urine culture and uroflowmetry, to assess flow peak (Qmax) and average flow (Qavg).

To assess changes in ejaculation, erection and urinary symptoms 3 validated questionnaires were used: the ICIQMLUTSsex, the IIEF-5 and the IPSS. Patients were evaluated preoperatively and 3 and 6 months postoperatively. Patients under alfa-blocker therapy were asked to suspend 
treatment 1 month before surgery, to assess the presence of preoperative ejaculation. PVR, Qmax and Qavg were also evaluated during follow-up.

The study was approved by our local Ethical Committee (approval $\mathrm{n}^{\circ}$ ASLMI2/201400327) and all patients signed the informed consent.

\section{Surgical technique}

The surgical technique is a modification of the original ThuLEP described by Herrmann et al. [6]. Instead of performing the initial inverted-U incision surrounding the veru montanum, the incision is shifted $1.5 \mathrm{~cm}$ cranial to the veru montanum. The incision is then extended towards the lateral lobes, leaving a small hill of prostatic tissue at the apex of the lateral lobes, each side of the veru montanum. After this tissue-sparing approach, the surgical procedure is performed as described by Herrmann, enucleating the median and lateral lobes separately.

The Piranha Morcellator (Richard Wolf) was used at the end of the enucleation to remove the enucleated prostatic lobes.

All ES-ThuLEP procedures were performed by one surgeon (GB), with a previous experience of more than 1000 conventional ThuLEPs, using the $200 \mathrm{~W}$-Cyber-TM laser generator (Quanta System). A maximum power of $120 \mathrm{~W}$ was set for cutting and $35 \mathrm{~W}$ for coagulation.

\section{Statistical analysis}

Statistical analysis was carried out to assess patients' data and outcomes (Student's $t$ test, Chi-square test, and logistic regression analysis). All reported $p$ values were obtained with the two-sided exact method at the conventional 5\% significance level. Data were analyzed using the R software v.3.2.3 (by The R Foundation for Statistical Computing, Vienna, Austria), according to previously published guidelines for the reporting of statistics [26].

\section{Results}

Two hundred and eighty three patients were finally enrolled. Patients' preoperative characteristics are listed in Table 1. Intra and perioperative findings, complications and incidental findings are shown in Table 2. Follow-up parameters are shown in Table 3: significant improvement in IPSS, Qmax, Qavg and PVR were obtained at 3 and 6 months; no significant differences were observed between erectile function before and after surgery according to the IIEF-5; analysis of postoperative ICIQ-mLUTSsex showed 203 patients (71.7\%) with persistant ejaculation at 3 months and 219 patients (77.4\%) at 6 months from the procedure. No significant
Table 1 Preoperative characteristics

\begin{tabular}{ll}
\hline Age (years): mean $\pm \mathrm{SD}$ (range) & $64.21 \pm 9.74(52-75)$ \\
Prostate volume (cc): mean $\pm \mathrm{SD}$ (range) & $82.13 \pm 64.44(21-235)$ \\
Adenoma volume (cc): mean $\pm \mathrm{SD}$ (range) & $67.84 \pm 61.99(6-215)$ \\
PSA (ng/ml): mean $\pm \mathrm{SD}$ (range) & $3.6 \pm 3.1(0.5-13.8)$ \\
Qmax (ml/s): mean $\pm \mathrm{SD}$ (range) & $9.5 \pm 5.7(3.5-13.2)$ \\
Qavg (ml/s): mean $\pm \mathrm{SD}$ (range) & $4.8 \pm 2.3(2.5-8.1)$ \\
Voided volume (ml): mean $\pm \mathrm{SD}$ (range) & $184 \pm 101(79-302)$ \\
PVR (ml): mean $\pm \mathrm{SD}($ range) & $262 \pm 145(120-402)$ \\
Previous $\alpha$-blocker therapy: $n(\%)$ & $221(78.1 \%)$ \\
Previous $\alpha$-blocker $+5 \alpha$-reducatase inhibitor & $35(12.4 \%)$ \\
$\quad$ therapy: $n(\%)$ & $71(25.1 \%)$ \\
Previous TRUS biopsy: $n(\%)$ &
\end{tabular}

differences were observed between ejaculation and erection preservation in patients with different prostate volumes.

\section{Discussion}

Loss of ejaculation, due to either retrograde or dry ejaculation, has always been considered as the price to pay to achieve relief from LUTS and QoL improvement, both with medical treatment (alfa-blockers) and after surgery [12]. In recent years LEEP techniques have proved to be excellent alternatives to TURP and OP in terms of LUTS resolution, allowing to reduce morbidity and complications [7-10]. However, despite excellent functional outcomes, these procedures have not been able to avoid postoperative EjD [12, 13]. Data have shown that the impact on sexual functions is comparable between HoLEP and TURP and that ejaculation and orgasm perception are the two most impacted domains after these procedures [27]. Similarly to HoLEP, ThuLEP causes retrograde ejaculation, despite having a silghtly inferior relative risk compared to standard TURP $[28,29]$.

Reduced sexual satisfaction, due to the loss of ejaculation, could discourage men from undergoing surgery for $\mathrm{BPH}$, delaying treatment, causing worstening of symptoms and damage to the bladder detrusor muscle. Therefore, an ES approach would be the ideal option, especially for young and sexually active men. Based on evidence regarding ejaculation physiology, which has highlighted the key role of the supramontanal and pericollicular tissue and of the musculus ejaculatorius [20-22], modifications to classical endoscopic surgical techniques have been created to preserve these areas of tissue and ejaculation. In 1998 Ronzoni et al. presented a pilot ejaculation-preserving TURP on 45 patients with small prostates, by sparing more than $1 \mathrm{~cm}$ of prostatic urethra above the veru montanum. $80 \%$ of the patients maintained their ejaculation. To note, the bladder neck tissue, which in the past was thought to be fundamental for the ejaculatory function, 
Table 2 Intra and perioperative parameters, complications and incidental findings

\begin{tabular}{ll}
\hline Intra and perioperative parameters & $81 \pm 62$ \\
Operative time (minutes): mean \pm SD & $90.7 \pm 45.3$ \\
Enucleated tissue weight (g): mean \pm SD & $24.2 \pm 16.8$ \\
Stop bladder irrigation (hours): mean \pm SD & $51 \pm 16.9$ \\
Catheter removal (hours): mean \pm SD & $3.6 \pm 2.8$ \\
Hospitalization (days): mean \pm SD & $2.89 \pm 1.72$ \\
Haemoglobin drop (g/dl): mean \pm SD & $4(1.4 \%)$ \\
Transfusions: $n(\%)$ & $6(2.1 \%)$ \\
Complications and incidental findings & $3(1.1 \%)$ \\
Acute urinary retention: $n(\%)$ & $1(0.4 \%)$ \\
Re-intervention for bleeding: $n(\%)$ & $1(0.4 \%)$ \\
Transitory postoperative incontinence: $n(\%)$ & $15(5.3 \%)$ \\
Bladder neck sclerosis: $n(\%)$ & $7(2.5 \%)$ \\
Pain/discomfort during ejaculation at 3 months: $n(\%)$ & $151(53.3 \%)$ \\
Pain/discomfort during ejaculation at 6 months: $n(\%)$ & $79(27.9 \%)$ \\
Haemospermia at 3 months: $n(\%)$ & $12(4.2 \%)$ \\
Haemospermia at 6 months: $n(\%)$ & $3(1.1 \%)$ \\
Incidental prostate adenocarcinoma: $n(\%)$ &
\end{tabular}

was entirely removed [23]. Alloussi et al. presented their modified ejaculation-preserving TURP (EP-TURP) on 86 patients, with a 60-month follow-up, which not only spared the supramontanal tissue, but also the paracollicular tissue. Significant IPSS, PVR and Qmax improvement were achieved, comparable to standard TURP and ejaculation was preserved in $90.8 \%$ of the patients [24]. Talab et al., instead, presented a video-article showing their EP-PVP: similarly to Alloussi et al. the supramontanal and paracollicular tissues were preserved; 160 patients were analyzed, with a maximum follow-up of 74 months; results seemed promising: significant improvement in voiding parameters was achieved and ejaculation was maintained in $86.6 \%$ of the patients [25].

Despite the different energy sources employed (electrocautery vs laser), preservation of the paracollicular and supramontanal tissues has proved to significantly increase ejaculation preservation, confirming the importance of the anatomical structures that have been described in these areas. Considering these encouraging results we modified classical ThuLEP as described above. We performed a prospective study on 283 patients, who underwent ES-ThuLEP, to investigate the impact of this technique on ejaculation, erection and micturition. Our series showed an overall conservation of antegrade ejaculation in $71.7 \%$ of the patients at 3 months, which improved to $77.4 \%$ at 6 months. We also performed a post hoc evaluation of ejaculation preservation in patients with different prostatic volumes $(<40 \mathrm{cc}, \geq 40$ and $\leq 100 \mathrm{cc}$ and $>100 \mathrm{cc}$ ), to evaluate whether large prostates could compromise the outcome of the procedure. No significant differences were found (Table 3), confirming that ES-ThuLEP allowed to effectively maintain ejaculation no matter what prostate size.

To the best of our knowledge, only one ES-LEEP technique has been presented before, by Kim et al. the Ejaculatory-hood HoLEP (EH-HoLEP) [30]. In their study 26 patients who underwent EH-HoLEP were compared to other 26 patients who underwent standard HoLEP. Ejaculation was maintained in $46.2 \%$ of the patients who underwent EH-HoLEP vs $26.9 \%$ of the patients who underwent conventional HoLEP. However, this difference was not statistically significant. The small number of patients analized by Kim et al. the difference in technique between EH-HoLEP and ES-ThuLEP and the different features between the holmium and thulium lasers could explain the better outcomes achieved with ES-ThuLEP and the other ES procedures. Similarly to other ES techniques, Kim et al. preserved $1 \mathrm{~cm}$ of tissue above the veru montanum, but only 2-3 $\mathrm{mm}$ of tissue around each side of it, with a radical enucleation of the prostatic apex. Whereas, with ES-ThuLEP two entire hills of tissue are preserved at the prostatic apex, each side of the veru montanum, together with $1.5 \mathrm{~cm}$ of tissue above the veru montanum. The importance of this wider tissue preservation is demonstrated in the video by Talab et al. [25], which shows the proximity of the ejaculatory ducts to the apical prostatic tissue. Sparing of this wider area of tissue at the prostatic apex, allows to better preserve the ejaculatory ducts and the muscular fibres surrounding them, which have an important role in the emission of semen and in 


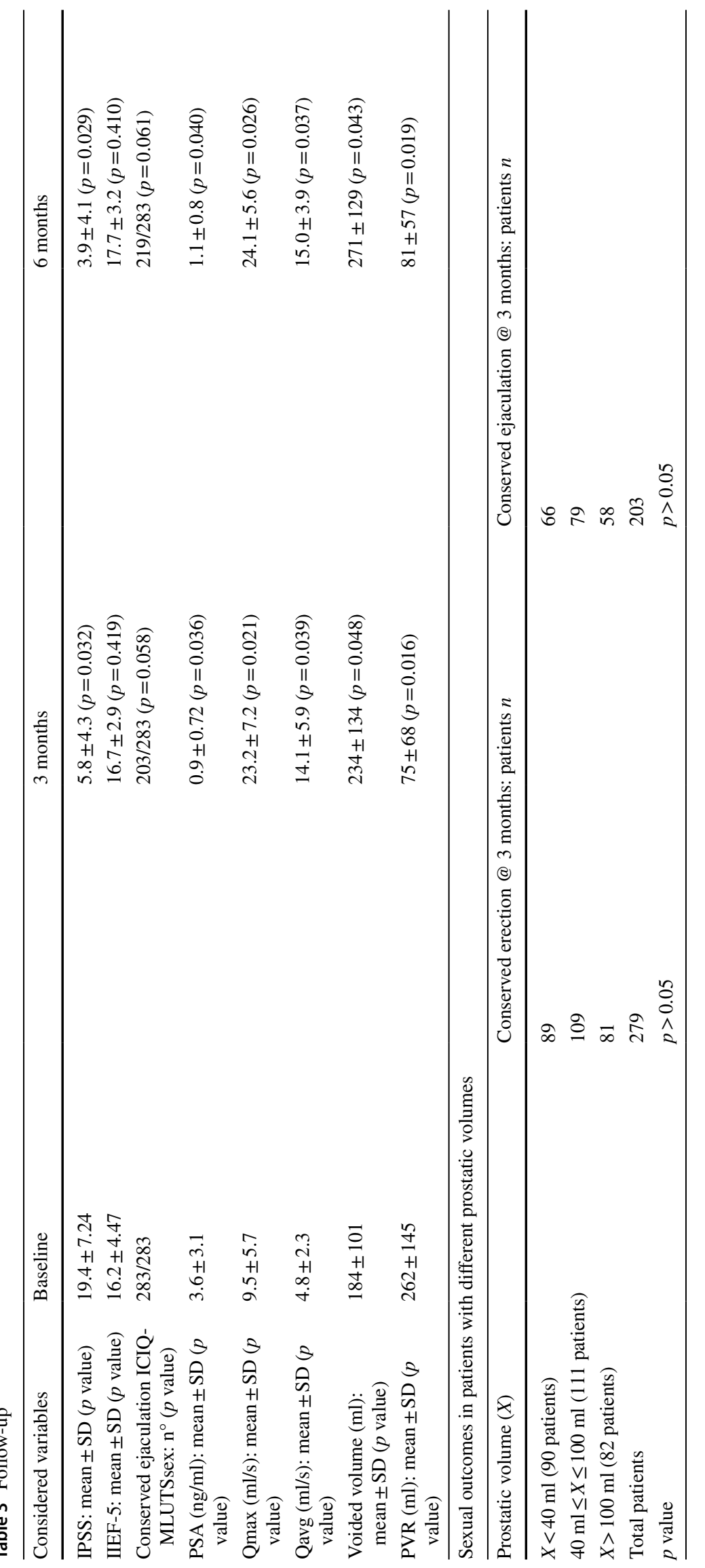


the conformational changes of the prostatic urethra during ejaculation [20-22]. The shallower penetration of the thulium laser compared to the holmium laser $(0.25 \mathrm{vs}$ $0.4 \mathrm{~mm}$ ) [31] and its continous wave output also allow to reduce the damage on these important structures and optimize their preservation. Whereas, the holmium laser, with its pulsed output, ruptures the tissue and more traction with the tip of the resectoscope is needed to separate the adenoma from the capsule, increasing mechanical stress on the surrounding tissues.

Differences in ejaculation-preserving rates could also be due to different surgical experiences and surgical volume. In this study the surgeon (GB) had previously performed more than 1000 classical ThuLEPs, which makes the surgeon very familiar with the equipment, the technique and the surgical landmarks. However, the other cited studies did not report previous surgical experience.

With regard to micturition improvement, we registered a mean IPSS of $5.8 \pm 4.3(p=0.032)$ at 3 months and $3.9 \pm 4.1(p=0.029)$ at 6 months. Qmax improved to $23.2 \pm 7.2 \mathrm{ml} / \mathrm{s}(p=0.021)$ and $24.1 \pm 5.6 \mathrm{ml} / \mathrm{s}(p=0.026)$ at 3 and 6 months, respectively (Table 3 ). This improvement is comparable to that registered in a previous study after standard ThuLEP, involving the same surgeon [28]. It is common thought amongst urologists, that leaving tissue at the prostatic apex means not disobstructing patients completely. The improvement in postoperative IPSS, flowmetry parameters and PSA that were registered in our study (Table 3), contradict this way of thinking and indicate that ES-ThuLEP allows to achieve significant micturition and LUTS improvement. This could be related to the fact that, most of the obstruction in $\mathrm{BPH}$, is secondary to the contraction of the prostatic smooth muscle fibres and to the pressure of the growing hyperplastic tissue on the prostatic capsule, which is directly transmitted to the prostatic urethra.

No changes in erectile function were observed during follow-up according to the IIEF-5 (Table 3), similarly to previously reported results for classical ThuLEP [28]. ES-ThuLEP also proved to be safe, with only 3 cases of postoperative haematuria requiring re-intervention, 6 cases of acute urinary retention (treated with bladder catheterization for 10 days and steroid oral therapy), 1 case of bladder neck sclerosis (treated with laser vaporization of the bladder neck) and 1 case of postoperative transitory incontinence, which was resolved at the 3-month follow-up (Table 2). At the 3-month follow-up 15 patients $(5.3 \%)$ reported occasional pain/discomfort during ejaculation, which reduced to $7(2.5 \%)$ at the 6-month followup; 151 patients $(53.3 \%)$ reported occasional haemospermia at the 3-month follow-up and 79 patients (27.9\%) at the 6-month follow-up. These findings are not uncommon in patients after BPH surgery. However, most studies do not report these individual outcomes, generally speaking of postoperative ejaculatory dysfunction, making it difficult to compare these findings.

The main limits of this study are the absence of a control group and its short follow-up. However, the primary outcome of the study was to assess the efficacy of this modified technique in preserving patients' ejaculation; therefore, a long follow-up was not planned. Future controlled trials will allow to better determine the ejaculation-preserving rate of this technique compared to standard ThuLEP. A longer follow-up, also evaluating re-intervention rate, will allow to understand if regrowth of the preserved apical tissue could be responsible for deterioration in micturition and LUTS. Nevertheless, considering the large amount of patients involved, our results seem promising, allowing to give patients, who wish to maintain ejaculation, a chance.

\section{Conclusions}

ES-ThuLEP allows to effectively preserve ejaculation in more than two thirds of the patients, no matter what prostate size, without compromising micturition improvement or erectile function and maintaining a low complication rate, making it a valid treatment alternative for young and sexually active men suffering from BPH.

Author contributions Protocol/project development: GB, LB, UB, CB, AC, MM, SM, BR. Data collection or management: GB, PB, RL, MM, ALP, MCS. Data analysis: GB, RL, ALP, MCS. Manuscript writing/ editing: GB, LB. Execution of surgical procedures: GB. Supervision: $\mathrm{GB}, \mathrm{CB}, \mathrm{SM}, \mathrm{AM}, \mathrm{BR}$.

Funding The authors declare that no extra institutional funding was received.

\section{Compliance with ethical standards}

Conflict of interest The authors declare that they have no conflict of interest or any known competing financial interests.

Ethical approval The study was performed in accordance with the ethical standards of the institutional and/or national research committee and with the 1964 Helsinki Declaration and its later amendments or comparable ethical standards. The study was approved by our local Ethical Commitee.

Informed consent Informed consent was obtained from all individual participants included in the study.

Consent for publication All patients gave their consent for the publication of this article. 


\section{References}

1. Lee SWH, Chan EMC, Lai YK (2017) The global burden of lower urinary tract symptoms suggestive of benign prostatic hyperplasia: a systematic review and meta-analysis. Sci Rep 7(1):7984

2. Rosen R, Altwein J, Boyle P et al (2003) Lower urinary tract symptoms and male sexual dysfunction: the multinational survey of the aging male (MSAM-7). Eur Urol 44(6):637-649

3. Naspro R, Gomez Sancha F, Manica M et al (2017) From "gold standard" resection to reproducible "future standard" endoscopic enucleation of the prostate: what we know about anatomical enucleation. Minerva Urol Nefrol 69(5):446-458

4. Herrmann TR (2016) Enucleation is enucleation is enucleation is enucleation. World J Urol 34(10):1353-1355

5. Gilling PJ, Kennett K, Das AK et al (1998) Holmium laser enucleation of the prostate (HoLEP) combined with transurethral tissue morcellation: an update on the early clinical experience. J Endourol 12(5):457-459

6. Herrmann TR, Bach T, Imkamp F et al (2010) Thulium laser enucleation of the prostate (ThuLEP): transurethral anatomical prostatectomy with laser support. Introduction of a novel technique for the treatment of benign prostatic obstruction. World J Urol 28(1):45-51

7. Bozzini G, Seveso M, Melegari S et al (2017) Thulium laser enucleation (ThuLEP) versus transurethral resection of the prostate in saline (TURis): a randomized prospective trial to compare intra and early postoperative outcomes. Actas Urol Esp 41(5):309-315

8. Sun F, Sun X, Shi Q et al (2018) Transurethral procedures in the treatment of benign prostatic hyperplasia: a systematic review and meta-analysis of effectiveness and complications. Medicine (Baltimore) 97(51):e13360

9. Lin Y, Wu X, Xu A et al (2016) Transurethral enucleation of the prostate versus transvesical open prostatectomy for large benign prostatic hyperplasia: a systematic review and meta-analysis of randomized controlled trials. World J Urol 34(9):1207-1219

10 Zhang J, Ou Z, Zhang X et al (2019) Holmium laser enucleation of the prostate versus thulium laser enucleation of the prostate for the treatment of large-volume prostates $>80 \mathrm{ml}$ : 18-month follow-up results. World J Urol. https://doi.org/10.1007/s00345-019-02945 $-\mathrm{x}$

11. Gravas S, Cornu JN, Gacci M et al (2019) EAU guidelines on management of non-neurogenic male lower urinary tract symptoms (LUTS) incl. benign prostatic obstruction (BPO). EAU Guidelines Office, Arnhem, The Netherlands. https://uroweb.org/ guideline/treatment-of-non-neurogenic-male-luts/

12. DeLay KJ, Nutt M, McVary KT (2016) Ejaculatory dysfunction in the treatment of lower urinary tract symptoms. Transl Androl Urol 5(4):450-459

13. Marra G, Sturch P, Oderda M et al (2016) Systematic review of lower urinary tract symptoms/benign prostatic hyperplasia surgical treatments on men's ejaculatory function: time for a bespoke approach? Int J Urol 23(1):22-35

14. Woo HH, Bolton DM, Laborde E et al (2012) Preservation of sexual function with the prostatic urethral lift: a novel treatment for lower urinary tract symptoms secondary to benign prostatic hyperplasia. J Sex Med 9(2):568-575

15. Albisinni $S$, Aoun $F$, Roumeguère $T$ et al (2017) New treatment strategies for benign prostatic hyperplasia in the frail elderly population: a systematic review. Minerva Urol Nefrol 69(2):119-132
16. Gratzke C, Barber N, Speakman MJ et al (2017) Prostatic urethral lift vs transurethral resection of the prostate: 2-year results of the BPH6 prospective, multicentre, randomized study. BJU Int 119(5):767-775

17. Lebdai S, Chevrot A, Doizi S et al (2019) Do patients have to choose between ejaculation and miction? A systematic review about ejaculation preservation technics for benign prostatic obstruction surgical treatment. World J Urol 37(2):299-308

18 Marberger H (1974) The mechanisms of ejaculation. Basic Life Sci 4(PT. B):99-110

19. Gallizia P (1972) The smooth sphincter of the vesical neck, a genital organ. Urol Int 27(4):341-354

20. Sturch P, Woo HH, McNicholas T et al (2015) Ejaculatory dysfunction after treatment for lower urinary tract symptoms: retrograde ejaculation or retrograde thinking? BJU Int 115(2):186-187

21. Gil-Vernet JM Jr, Alvarez-Vijande R, Gil-Vernet A et al (1994) Ejaculation in men: a dynamic endorectal ultrasonographical study. Br J Urol 73(4):442-448

22. Dorschner W, Stolzenburg JU (1994) A new theory of micturition and urinary continence based on histomorphological studies. 3. The two parts of the musculus sphincter urethrae: physiological importance for continence in rest and stress. UrolInt 52(4):185-188

23. Ronzoni G, De Vecchis M (1998) Preservation of anterograde ejaculation after transurethral resection of both the prostate and bladder neck. Br J Urol 81(6):830-833

24. Alloussi SH, Lang C, Eichel R et al (2014) Ejaculation-preserving transurethral resection of prostate and bladder neck: short- and long-term results of a new innovative resection technique. $\mathrm{J}$ Endourol 28(1):84-89

25. Talab SS, Santiago-Lastra YA, Bachmann A et al (2013) The impact of ejaculation-preserving photo-selective vaporization of the prostate (EP-PVP) on lower urinary tract symptoms and ejaculatory function: results of a multicenter study. J Urol 189(4):e164

26. Vickers AJ, Sjoberg DD (2015) Guidelines for reporting of statistics in European urology. EurUrol 67:181-187

27. Briganti A, Naspro R, Gallina A et al (2006) Impact on sexual function of holmium laser enucleation versus transurethral resection of the prostate: results of a prospective, 2-center, randomized trial. J Urol 175(5):1817-1821

28. Carmignani L, Bozzini G, Macchi A et al (2015) Sexual outcome of patients undergoing thulium laser enucleation of the prostate for benign prostatic hyperplasia. Asian J Androl 17(5):802-806

29. Cacciamani GE, Cuhna F, Tafuri A et al (2019) Anterograde ejaculation preservation after endoscopic treatments in patients with bladder outlet obstruction: systematic review and pooled-analysis of randomized clinical trials. Minerva Urol Nefrol 71(5):427-434

30. Kim M, Song SH, Ku JH et al (2015) Pilot study of the clinical efficacy of ejaculatory hood sparing technique for ejaculation preservation in Holmium laser enucleation of the prostate. Int J Impot Res 27(1):20-24

31. Gravas S, Bachmann A, Reich O et al (2011) Critical review of lasers in benign prostatic hyperplasia (BPH). BJU Int 107(7):1030-1043

Publisher's Note Springer Nature remains neutral with regard to jurisdictional claims in published maps and institutional affiliations. 